\title{
Genetic Diversity Analysis and Phylogenetic Relationship among a Representative Collection of Spanish Pepper (Capsicum annuum) Landraces
}

\author{
Miguel BRACHO-GIL, Leandro PEREIRA-DIAS, Santiago VILANOVA, Ana FITA, \\ Jaime PROHENS, Adrián RODRÍGUEZ-BURRUEZO* \\ Instituto de Conservación y Mejora de la Agrodiversidad Valenciana (COMAV), Universitat Politècnica \\ de València, Camino de Vera 14, 46022 Valencia, Spain \\ *corresponding author: drodbur@doctor.upv.es
}

Bulletin UASVM Horticulture 75(1) / 2018

Print ISSN 1843-5262, Electronic ISSN 1843-536X

DOI:10.15835/buasvmcn-hort: 000717

\begin{abstract}
Spain is one of the most important centers of diversity for Capsicum annuum peppers, and a plethora of ecotypes can be found throughout the country. These resources represent a valuable genetic pool which must be characterized in depth both morphologically and molecularly. The aim of the present work was to assess phenotypic and genotypic diversity and relationships within some Spanish pepper landraces. A collection of 12 Spanish pepper landraces and 15 foreign controls was characterized with a set of 46 descriptors from International Plant Genetic Resources Institute (IPGRI, now Bioversity International) and genotyped using Genotyping-by-Sequencing (GBS). The studied collection was genetically diverse. The morphological characterization allowed discrimination of the varieties through fruit size, growth habit and others traits. SNP-based phylogenetics clustered together Spanish accessions. Our results provide useful information of several different ecotypes to boost breeding programs.
\end{abstract}

Keywords: morphological characterization, single nucleotide polymorphism, paprika landraces

\section{Introduction}

Chile peppers and paprika (Capsicum spp.) are one of the most important vegetables and spices in the world (FAO, 2014). They are used in a range of dishes and recipes, being $C$. аnnuum the most common and diverse species (DeWitt and Bosland, 2009). Spain was for a long time the entrance to a considerable genetic flow from the Americas, which explains the great amount of Spanish varieties and landraces nowadays (Rodríguez-Burruezo et al., 2016). These resources represent a valuable genetic pool (Lanteri, 2005; Nicolaï, 2012). In order to optimize their use by breeders, these materials must be characterized in depth both morphologically and molecularly. The combination of these two characterization strategies may produce molecular markers correlated to relevant agronomic traits. New technologies like GBS enable the generation of massive raw data that leads to large sets of SNPs
(Single Nucleotide Polymorphism). The aim of the present work was to assess phenotypic and genotypic diversity and relationships within Spanish pepper landraces.

\section{Materials and methods}

Plant material consisted on an encompassing collection of 12 C. annuum accessions from different origins within Spain, as well as 15 non-Spanish control accessions that represent the variability of the species (Tab. 1). The collection was initially characterized with a set of 46 IPGRI descriptors (IPGRI, 1995) (Tab. 2), ANOVA and Principal Component Analysis was performed to identify significant differences among accessions. DNA was extracted from one plant per genotype by modified Doyle and Doyle method (1990). Afterwards, Genotyping-by-Sequencing (GBS) methodology described by Elshire et al. (2011) was followed to genotype the whole collection. Consequently SNP- 
Table 1. Plant material. Spanish landraces and foreign controls

\begin{tabular}{|c|c|c|c|}
\hline Name & Code & Origin & Fruit type (IPGRI) \\
\hline \multicolumn{4}{|c|}{ Spanish landraces } \\
\hline Arnoia & Arn & Galicia, Spain & Blocky \\
\hline Bola DOP & Bol & Murcia, Spain & Almost round \\
\hline Calahorra & Cal & La Rioja, Spain & Blocky \\
\hline Guernika & Guer & País Vasco, Spain & Elongated \\
\hline Guindilla Ibarra & Iba & País Vasco, Spain & Elongated \\
\hline Largo de Reus & Reus & Tarragona, Spain & Blocky \\
\hline M. Fresno de la Vega & Fres & León, Spain & Blocky \\
\hline Najerano & $\mathrm{Naj}$ & La Rioja, Spain & Triangular \\
\hline Padrón & Pad & Galicia, Spain & Blocky \\
\hline Pimiento del Bierzo IGP & Bie & León, Spain & Blocky \\
\hline Piquillo IGP & Piq & Navarra, Spain & Triangular \\
\hline Valenciano & VLC & Valencia, Spain & Blocky \\
\hline \multicolumn{4}{|c|}{ Foreign control accessions } \\
\hline Ací Sivri & Aci & Turkey & Elongated \\
\hline Ancho 101 & 101 & Mexico/USA & Triangular \\
\hline Ancho mulato & Mul & Mexico & Triangular \\
\hline Chile de Arbol & Árb & Mexico & Elongated \\
\hline Chile Serrano & Serr & Mexico & Triangular \\
\hline Chimayo & Chim & New Mexico, USA & Blocky \\
\hline Di Senise IGP & Sen & Potenza, Italy & Elongated \\
\hline Jalapeño $M$ & Jal & Mexico & Elongated \\
\hline Numex Big Jim & Bjim & New Mexico, USA & Elongated \\
\hline Pasilla Bajío & Baj & Mexico & Elongated \\
\hline Petit Marsellais & Pet & France & Blocky \\
\hline Poivre Rouge de Bresse & PRB & France & Elongated \\
\hline Tendre Châteaurenard & Chat & France & Blocky \\
\hline Torpedo Bangalore & Ban & India & Elongated \\
\hline Habanero rojo (C. chinense) & Haba & Pensilvania, USA & Campanulated \\
\hline
\end{tabular}

Table 2. Capsicum IPGRI descriptors

\begin{tabular}{|c|c|c|c|}
\hline \multicolumn{4}{|c|}{ Plant } \\
\hline Stem coour & Stem shape & Stem pubescence & Nodal anthocyanin \\
\hline Leaf colour & Leaf shape & Leaf pubescence & Lamina margin \\
\hline Mature leaf length & Mature leaf width & & \\
\hline \multicolumn{4}{|c|}{ Inflorescence } \\
\hline Days to flowering & Number of flowers per axil & Flower position & Corolla colour \\
\hline Corolla spot colour & Corolla shape & Corolla length & Anther colour \\
\hline Anther length & Filament colour & Filament length & Stigma exsertion \\
\hline Male sterility & Calyx Pigmentation & Calyx margin & Calyx annular constriction \\
\hline \multicolumn{4}{|c|}{ Fruit } \\
\hline Days to fruiting & Anthocyanin spots & $\begin{array}{c}\text { Fruit color at intermediate } \\
\text { stage }\end{array}$ & Fruit color at mature stage \\
\hline Shape & Length & Width & Weight \\
\hline Pedicel length & Fruit wall thickness & $\begin{array}{l}\text { Shape at pedicel } \\
\text { attachment }\end{array}$ & Neck at base of fruit \\
\hline Fruit shape at blossom end & $\begin{array}{l}\text { Fruit blossom end } \\
\text { appendage }\end{array}$ & $\begin{array}{l}\text { Fruit cross-sectional } \\
\text { corrugation }\end{array}$ & Number of locules \\
\hline $\begin{array}{l}\text { Ripe fruit pedicel with fruit } \\
\text { persistance }\end{array}$ & $\begin{array}{l}\text { Ripe fruit pedicel with } \\
\text { stem persistance }\end{array}$ & Fruit surface & Placenta length \\
\hline
\end{tabular}



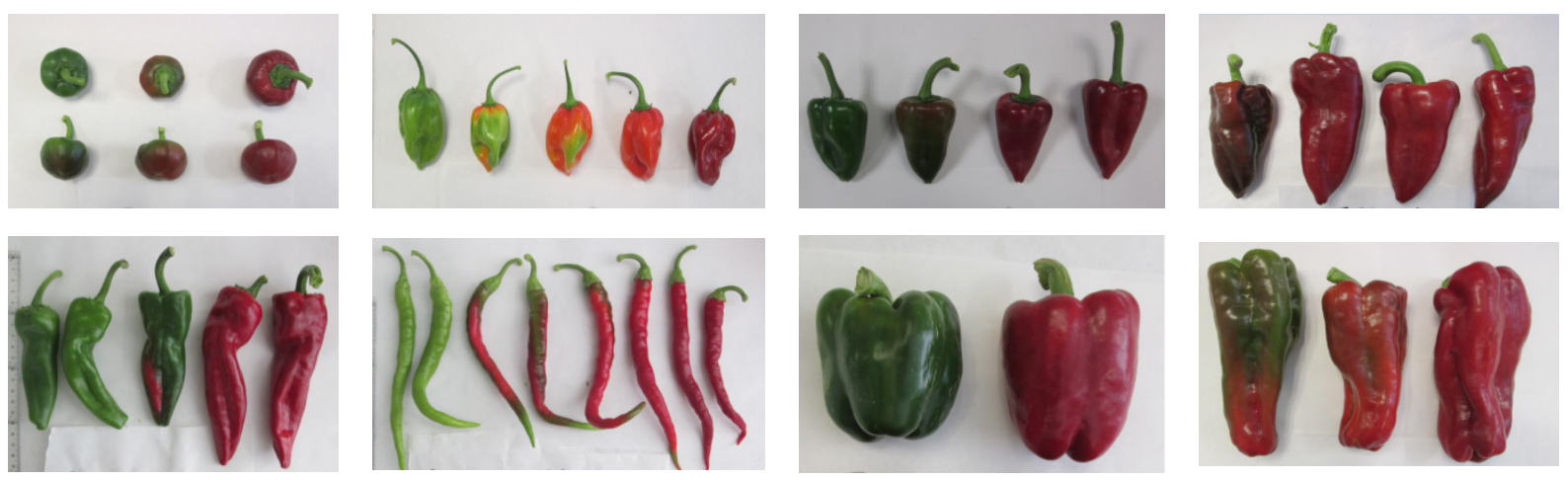

Figure 1. Fruit varietal types. Left to right and top to bottom: Almost round: Bola; Campanulated: Habanero rojo; Triangular: Piquillo, Najerano; Elongated: Guernika, Guindilla Ibarra; Blocky: Morrón Fresno de la Vega, Largo de

Reus

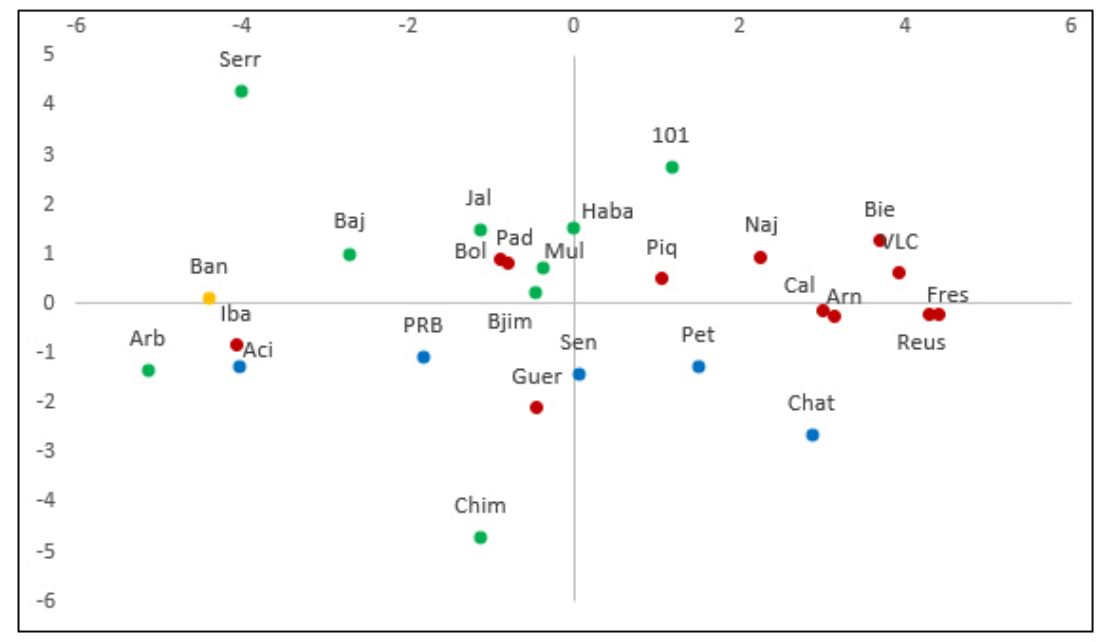

Figure 2. Varieties distribution based on analysis of principal component 1 (27.4\%) and principal component 2 $(10.2 \%)$. On the left side of the graph are smaller, elongated varieties (Iba, Bjim, Baj), while on the right side there are blocky and bigger landraces (Naj, Reus, VLC).

calling was performed using Glaubitz et al. (2014) protocol. Finally, phylogenetic analysis was done relying on SNP-based genetic distances to assess the degree of relatedness between genotypes, using SNPhylo (Lee et al., 2014).

\section{Results and discussion}

The morphological characterization allowed discrimination of the varieties through principal component analysis (Fig. 2). Out of the initial 46 IPGRI descriptors, 36 showed significant differences. The first two components explain $27.4 \%$ and $10.2 \%$ of total variability. Several traits such as fruit shape and size, plant height and growth habit, showed statistical difference for several landraces (data not shown). On the PCA, varieties grouped mainly based on shape. On the basis of the genetic distance matrix a phylogenetic tree was constructed. Landraces of the same origin clustered together (Fig. 3). Even though there are similarities within certain landraces, the whole collection was found to be phenotypically and genetically diverse, which supports the initial assessment of Spanish pepper landraces as invaluable resources as breeding materials for future European pepper trade.

\section{Conclusion}

Our results provide useful information of several different ecotypes to boost breeding 


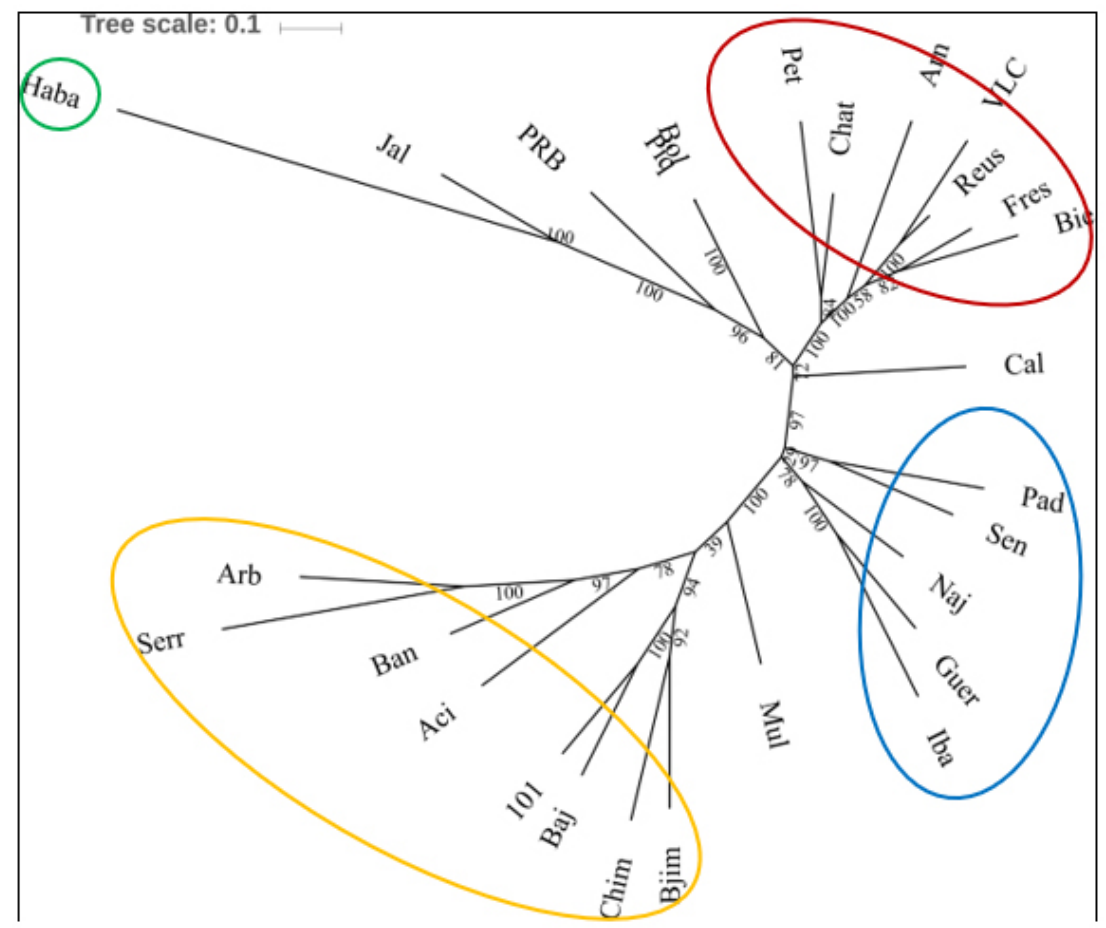

Figure 3. Phylogenetic tree based on genetic distance. Circled: C. chinense control Habanero (green); Mexican/ U.S. varieties (yellow); Blocky-Spanish/European landraces (red); Elongated-Spanish/European varieties (blue).

programs, germplasm management and conservation of landraces of C. annuum in Spain.

Acknowledgements. This work was partly financed by projects RTA2013-00022-C02-02 and RTA2014-00041-C02-02, funded by the Inst. Nacional Investigaciones Agrarias (Spain), FEDER funds.

\section{References}

1. Dewitt D, Bosland PW (2009). The complete chile pepper book: A gardener's guide. Timbre Press, Portland, Oregon, USA.

2. Elshire RJ, Glaubitz JC, Sun Q, Poland JA, Kawamoto K, Buckler ES, Mitchell SE (2011). A Robust, Simple Genotyping-by-Sequencing (GBS) Approach for High Diversity Species. PLoS ONE 6(5): e19379. doi:10.1371/ journal.pone.0019379

3. Food and Agriculture Organization of the United Nations (2017). FAOSTAT Statistics Database.
4. Glaubitz JC, Casstevens TM, Lu F, Harriman J, Elshire RJ, Sun Q, Buckler ES (2014). TASSEL-GBS: A High Capacity Genotyping by Sequencing Analysis Pipeline. PLoSONE 9(2): e90346. doi:10.1371/journal.pone.0090346

5. IPGRI, AVRDC and CATIE (1995). Descriptors fo Capsicum (Capsicum spp.) International Plant Genetic Resources Institute, Rome, Italy; The Asian Vegetable Research and Development Center, Taipei, Taiwan; Centro Agronómico Tropical de Investigación y Enseñanza, Trrialba, Costa Rica.

6. Lee TH, Guo H, Wang X, Kim C, Paterson AH (2014). SNPhylo: a pipeline to construct a phylogenetic tree from huge SNP data. BMC Genomics, 15(1).

7. Rodríguez-Burruezo A, Pereira L, Fita A (2016). 21. Pimiento. In: Las variedades locales en la mejora genética de plantas (Eds. Ruiz de Galarreta, Prohens J and Tierno R). Servicio central de Publicaciones del Gobierno Vasco, Spain. (pp. 405-426). 